\title{
Physicians may require heroic efforts to enable survival in acute pulmonary embolism due to socioeconomic disparities in access to surgical care
}

\author{
Toktam Alirezaei and Behzad Hajimoradi \\ Department of Cardiology, Shohadaye-Tajrish Hospital, Shahid Beheshti University of Medical Sciences, Tehran, Iran
}

Venous thromboembolism is one of the big three cardiovascular diseases and a leading cause of morbidity and mortality worldwide $[1,2]$.

In the last decades, many efforts have been made to improve the overall health and eliminate disparities in healthcare delivery among different socioeconomic status groups.

Practical design guidelines produce a significant progression in diagnostic, therapeutic, and preventive strategies of Pulmonary embolism (PE) [3]. Despite these advances, the increased incidence of $\mathrm{PE}$ and patients requiring advanced therapy has made new challenging.

Low-risk and sub massive PE are more common, constitute the majority of PE cases and their treatment strategies are clear [4,5]. Patients with massive PE account for $5-10 \%$ of total PEs which can develop cardiogenic shock culminating in multisystem organ failure and have a high mortality rate (would be informative to have mortality rates with massive and sub massive PEs, with and/or without appropriate treatment). These patients require advanced therapies including systemic thrombolysis, pharmacomechanical catheterdirected therapy, surgical embolectomy [6,7].

The guidelines of the American Heart Association and the guidelines of the American College of Chest Physicians recommended that "patients with massive pulmonary embolism, evidence of hemodynamic compromise, and acceptable bleeding risk receive a thrombolytic agent" $[8,9]$.

Despite the use of thromboprophylaxis and advances in treatment strategies, the number of patients who need invasive therapeutic procedures, e.g. surgical intervention, are increasing. For example, in recent years, owing to remarkable improvements in the field of treatment of metastatic cancers, long-term survival can be achieved in many patients [10]. An ongoing neoplasm and acute PE create challenges for management due to increased risk of bleeding following thrombolysis as well especially in case of brain metastasis $[11,12]$.

Based on guideline recommendations, catheter embolectomy with fragmentation or surgical embolectomy is indicated in patients with significant massive PE who have contraindications to fibrinolysis or who remain unstable after receiving fibrinolysis $[8,9]$.

It is proportionately easy to draw up guidelines for management of massive PE in ideal services in high-income economies, which could never be achieved in practice in low- and middle-income economies, as regards disparities between different economic strata in societies appear to be growing rather than shrinking.
Agarwal et al. [13] reported that the place of residence in the Unites States might play a major role in influencing the outcome following acute $\mathrm{PE}$ and there was a significant difference in the inhospital mortality rates and receive thrombolysis between the highest and lowest socioeconomic status patients diagnosed with acute PE. In addition, other studies showed disparities in treatment strategies and mortality rates for ischemic stroke in the Unites States due to socioeconomic, racial and ethnic disparities. While disparities between different socioeconomic strata in a single country are significant, hence, they can be more significant between high income country and low- middle income country.

It can be hypothesized that disparities in outcomes following acute $\mathrm{PE}$ in different income countries might be mediated by differences in factors such as biologic risk factors, comorbidities, a delay in recognition of symptoms or in presentation to the hospital among patients, but they also might be related to unique aspects of disparities in the utilization or availability of treatments like catheter or surgical embolectomy in health care centers. This serves to provide an insight into the important impact of disparities on treatment strategies of acute PE.

It is common for clinicians in low and middle economic countries to be confronted with the dilemma of how to manage patients admitted with massive PE in life-threatening settings who are at extremely high risk for bleeding complications of thrombolysis and no other acceptable recourse such as catheter or surgical embolectomy was available and accessible [12,14].

What does the clinician do? There are different strategies that may be used.

The clinician may think about the choices beyond the guidelines limits and give the thrombolytic agent as a last resort to save a life which do not meet guidelines or due to fear of bleeding risk may continue conservative medical treatment that appears to be failed in this critical setting.

There is lack of a clear guideline for how clinicians should manage an acute massive PE in the presence of contraindication to fibrinolysis while catheter and surgical embolectomy are not available or due to

${ }^{\star}$ Correspondence to: Toktam Alirezaei, Department of Cardiology, ShohadayeTajrish Hospital, Shahid Beheshti University of Medical Sciences, Tehran, Iran, E-mail: alirezaei.toktam@sbmu.ac.ir

Received: December 02, 2018; Accepted: December 14, 2018; Published: December 21, 2018 
high patient risk the surgeon refuses to perform the procedure, which leads to a large dilemma.

This is the fact that socioeconomic disparities in access to treatments of acute PE exist and it is one of the dilemmas facing clinicians in lowand middle-income countries.

The mission of clinicians is to provide lifesaving therapies to as many patients as possible, so, they may be forced to decide beyond the guidelines.

\section{References}

1. Wendelboe AM, Raskob GE (2016) Global Burden of Thrombosis: Epidemiologic Aspects. Circ Res 118: 1340-1347. [Crossref]

2. Jiménez D, de Miguel-Díez J, Guijarro R, Trujillo-Santos J, Otero R, et al. (2016) Trends in the Management and Outcomes of Acute Pulmonary Embolism: Analysis from the RIETE Registry. J Am Coll Cardiol 67: 162-170. [Crossref]

3. Smith SB, Geske JB, Kathuria P, Cuttica M, Schimmel DR, et al. (2016) Analysis of National Trends in Admissions for Pulmonary Embolism. Chest 150: 35-45. [Crossref]

4. Konstantinides SV, Torbicki A, Agnelli G, Danchin N, Fitzmaurice D, et al. (2014) 2014 ESC guidelines on the diagnosis and management of acute pulmonary embolism. Eur Heart J 35: 3033-3069, 3069a-3069k. [Crossref]

5. Stein PD, Matta F, Hughes MJ (2016) Home Treatment of Deep Venous Thrombosis According to Comorbid Conditions. Am J Med 129: 392-397. [Crossref]

6. Dalen JE (2002) Pulmonary embolism: what have we learned since Virchow?: treatment and prevention. Chest 122: 1801-1817. [Crossref]
7. Keeling WB, Sundt T, Leacche M, Okita Y, Binongo J, et al. (2016) Outcomes After Surgical Pulmonary Embolectomy for Acute Pulmonary Embolus: A Multi-Institutional Study. Ann Thorac Surg 102: 1498-1502. [Crossref]

8. Kearon C, Kahn SR, Agnelli G, Goldhaber S4, Raskob GE, et al. (2008) Antithrombotic therapy for venous thromboembolic disease: American College of Chest Physicians Evidence-Based Clinical Practice Guidelines (8thEdition). Chest 133:454-545. [Crossref]

9. Kucher N, Goldhaber SZ (2005) Management of massive pulmonary embolism. Circulation 112: e28-32. [Crossref]

10. Hisada Y, Geddings JE, Ay C, Mackman N, et al. (2015) Venous thrombosis and cancer: from mouse models to clinical trials. J Thromb Haemost 13: 1372-1382. [Crossref]

11. Tabouret E, Chinot O, Metellus P, Tallet A, Viens P, et al. (2012) Recent trends in epidemiology of brain metastases: an overview. Anticancer Res 32: 4655-4662. [Crossref]

12. Alirezaei T, Hajimoradi B, Pishgahi M, Nekooghadam SM, Golmohamadi M (2018) Successful systemic thrombolytic therapy for massive pulmonary embolism in a patient with breast cancer, brain metastasis and thrombocytopenia: A Case Report. Clin Case Rep 6: 1431-1435. [Crossref]

13. AgarwalS, Menon V, Jaber WA (2015) Residential zip code influences outcomes following hospitalization for acute pulmonary embolism in the United States. Vasc Med 20: 439-446. [Crossref]

14. Pishgahi M, Alirezaei T, Hajimoradi B, Nekooghadam SM, Shahi S (2018) Systemic fibrinolytic therapy in the presence of absolute contraindication; a case series. Emerg (Tehran) 6: e25. [Crossref]

Copyright: $(02018$ Alirezaei T. This is an open-access article distributed under the terms of the Creative Commons Attribution License, which permits unrestricted use, distribution, and reproduction in any medium, provided the original author and source are credited. 